\title{
Alcances y limitaciones de los mecanismos de coordinación gubernamental en la política anticorrupción: el caso de la Oficina de Fortalecimiento Ético y Lucha contra la Corrupción en Sunat ${ }^{+}$
}

\author{
CHRISTIAN CHIROQUE RUIZ* \\ Pontificia Universidad Católica del Perú \\ christian.chiroque@pucp.edu.pe
}

https://doi.org/10.18800/rcpg.201702.005

\section{Resumen}

Desde mediados del año 2000, salió a la luz una compleja red de corrupción que capturó a diversas entidades del Estado en la década de 1990, lo que generó el surgimiento de una serie de iniciativas de política vinculadas con la lucha contra la corrupción. Durante ese periodo, la Superintendencia Nacional de Aduanas y de Administración Tributaria (Sunat) fue una de las instituciones que estuvo inmersa en casos de gran corrupción, asimismo se ha constituido como una entidad con alto riesgo en la incidencia de casos de corrupción hasta la actualidad. Por ello, desde 2012 se puso en funcionamiento la Oficina de Fortalecimiento Ético y Lucha contra la Corrupción, la cual tiene por objetivo el desarrollo de acciones de prevención y control de la corrupción dentro de los procesos institucionales de la institución. El presente artículo tiene como objetivo identificar los mecanismos de coordinación gubernamental usados en la implementación de políticas anticorrupción dentro de la Sunat, durante el periodo 2012-2015. Asimismo, se busca describir cuáles fueron las principales limitaciones que se presentaron durante dicho proceso. Según los resultados de la investigación, entre las principales limitaciones se encuentran: (i) la influencia política de la alta dirección durante la investigación de casos de corrupción, (ii) el solapamiento de distintos dispositivos de política anticorrupción, (iii) vacíos dentro del funcionamiento del Órgano de Control Institucional (OCI), (iv) ineficientes sistemas de identificación de riesgos de corrupción, (v) desiguales capacidades técnicas entre las áreas encargadas de la política, (vi) así como limitados recursos logísticos y presupuestales.

Palabras clave: mecanismos de coordinación gubernamental, política anticorrupción, riesgo de corrupción, aduanas, Sunat.

+ Recibido el 23 de marzo de 2017; aceptado el 8 de agosto de 2017.

* Licenciado en Ciencia Política y Gobierno y candidato a magíster en Gerencia Social, con mención en Gerencia de Programas y Proyectos de Desarrollo, por la Pontificia Universidad Católica del Perú. 


\title{
Uses and limitations of anti-corruption coordination mechanisms. The case of the Anticorruption and Ethics Office of Sunat
}

\begin{abstract}
In mid-2000, a complex network of corruption that captured various governmental entities during the last decade came to light. This prompted the subsequent emergence of a number of measures linked to the fight against corruption. During that period, the Superintendencia Nacional de Aduanas y de Administración Tributaria (Sunat) was one of the institutions immersed in cases of grand corruption, and at the present it is an entity with high vulnerability to the incidence of cases of petty corruption. For this reason, since 2012, the Oficina de Fortalecimiento Ético y Lucha contra la Corrupción (Oferlccor) has been set into operation. This institution has the objective to develop actions for prevention and control of corruption within various administrative processes of the entity. This article aims to identify the coordination mechanism used during the implementation of anticorruption policies in Sunat during the period 2012-2015. In addition, it seeks to describe what the main limitations that occurred during that process were. According to the research results, the main constraints were two: (i) the political influence of senior management during research processes, (ii) the overlapping of different policy devices, (iii) gaps in the operation of the Órgano de Control Institucional (OCI), (iv) inefficient risk identification systems, (v) unequal technical capacities between the areas involved in the policy, as well as (vi) limited logistical and budgetary resources.
\end{abstract}

Keywords: mechanisms of coordination, anti-corruption policy, risk of corruption, customs, Sunat. 


\section{INTRODUCCIÓN}

En el Perú, luego del régimen de Alberto Fujimori (1990-2000), surgieron una serie de iniciativas de lucha contra la corrupción, debido a las fatales consecuencias que tuvieron numerosos casos de corrupción que se presentaron en las altas esferas de poder gubernamental durante la década de los noventa. Desde entonces, la corrupción se ha convertido en uno de los problemas públicos de mayor relevancia dentro del sistema político peruano, por sus efectos perjudiciales al crecimiento económico, a la estabilidad política y la institucionalidad del país (Hussmann, 2007).

Dentro de algunos análisis, suele suponerse que las políticas anticorrupción siguen un proceso ordenado, en el cual el Estado posee control sobre el escenario y dispone de las capacidades institucionales y las herramientas necesarias para implementar y asegurar el logro de los objetivos de acuerdo al planeamiento y fines de la política pública. De modo general, el proceso de implementación de una política pública comprende la interacción de diversos actores, en distintos niveles y espacios, para llevar a cabo un curso de acción basado en lo que ha sido propuesto por alguna legislación, plan o programa gubernamental (Aguilar, 1993, p. 47). Así, las políticas anticorrupción son procesos particularmente complejos y multidimensionales, difíciles de gestionar, en los cuales no siempre es suficiente contar con un documento o decisión previa para asegurar su implementación.

Este artículo analiza el proceso de implementación de la política anticorrupción a través del estudio de caso de la Oficina de Fortalecimiento Ético y Lucha contra la Corrupción (Ofelccor) en la Superintendencia Nacional de Aduanas y de Administración Tributaria (Sunat). Dicha institución, durante los años noventa, estuvo inmersa en casos de gran corrupción y se posicionó como una entidad con alta sensibilidad y vulnerabilidad a la incidencia de casos de corrupción hasta el día de hoy. De esta manera, la Oferlccor tiene por objetivo el desarrollo de acciones de prevención y lucha contra la corrupción dentro de los procesos institucionales de la entidad.

El estudio posee una metodología cualitativa, enfocándose en la obtención de información respecto a tres objetivos específicos: a) describir el proceso de implementación de la política anticorrupción en Sunat, b) identificar los mecanismos de coordinación gubernamental implementados, y c) analizar las limitaciones de la coordinación y problemas operativos que se presentaron en la entidad para llevar a cabo dicha política.

En esta línea, se optó por centrar el trabajo de campo en la realización de entrevistas semiestructuradas a los principales actores involucrados en el proceso 
de implementación de la política anticorrupción en Sunat: funcionarios y exfuncionarios de la Oferlccor, así como de la Oficina de Control Institucional de la entidad, personal de las Intendencias de Aduanas, funcionarios de la Comisión de Alto Nivel Anticorrupción-PCM y especialistas en la materia. Asimismo, se utilizaron fichas de registro para sistematizar la información obtenida de la normativa y legislación vinculada a lucha contra la corrupción, así como documentos producidos por la PCM, CAN-Anticorrupción y Oferlccor.

La presente investigación se organiza en cuatro secciones. La primera comprende una discusión teórica en torno al concepto de coordinación gubernamental; la segunda detalla el proceso de la política anticorrupción en el periodo de estudio; la tercera describe el diseño institucional de la política anticorrupción en Sunat; y la última sección aborda el funcionamiento de la Oficina de Fortalecimiento Ético y Lucha contra la Corrupción (Ofelccor), la identificación de los mecanismos de coordinación utilizados, así como cuáles son las principales limitaciones para la implementación de este tipo de políticas públicas.

\section{ELEMENTOS CONCEPTUALES SOBRE COORDINACIÓN GUBERNAMENTAL}

Desde la década del setenta, los distintos estudios de políticas públicas hicieron un giro en el foco de análisis, el cual comenzó a poner mayor énfasis en la fase de implementación de políticas, mostrando así la necesidad de lograr mayor eficiencia en los resultados de las decisiones gubernamentales, que serían traducidas en políticas o programas específicos. Las políticas públicas son procesos complejos que requieren la intervención de múltiples actores con características distintas, los cuales deben de interactuar a fin de conseguir el objetivo de dicha política: la solución del problema público.

La coordinación o alineamiento entre los actores conlleva a una mayor eficiencia e integralidad en la ejecución de políticas públicas, lo cual aseguraría el logro de los resultados esperados (Martínez, 2010). En los últimos años, se ha constatado que la mayoría de problemas públicos son de carácter complejo y multidimensional, lo cual no permite su tratamiento segmentado o sectorial, debido a la gran cantidad de factores y escenarios en los que se pueden manifestar (Martínez, 2010, p. 14). Esta diversidad de escenarios origina la necesidad de que los actores tomen decisiones y asignen recursos a la implementación de una política pública en sus respectivas jurisdicciones particulares, sean estas sectoriales (Poder Ejecutivo) o territoriales (nacional, regional o local). Por consiguiente, la coordinación se convirtió en un tema relevante a tratar por académicos y funcionarios del Estado responsables de las políticas públicas, 
los cuales han devenido en un amplio conjunto de estudios que brindan diversas perspectivas desde donde se analiza el cada vez más importante papel de la coordinación en el logro de políticas públicas eficientes.

Un concepto ampliamente aceptado de coordinación es el postulado por Peters, que implica el estado en el cual las distintas organizaciones, públicas y privadas, responsables de la formación de políticas y los programas de gobierno trabajen juntas a fin de realizar acciones mínimamente redundantes, contradictorias o incoherentes (1998). Para el autor, un programa o política es más o menos coordinado si es que se evita la duplicación y superposición de decisiones y se reducen inconsistencias de las políticas. Además, se deben asegurar prioridades, a fin de apuntar a la cohesión y coherencia de ellas, de tal forma que se atenúe el conflicto político y burocrático. En general, se propone una perspectiva holística reduciendo la mirada sectorial y estrecha de las políticas.

No obstante, la coordinación puede tener distintos ámbitos. Desde estudios propios de la gestión pública, Painter (1981) destaca la diferencia entre coordinación política y coordinación administrativa. La coordinación política (plano de la decisión) se focaliza más en fines que en medios, mientras que la administrativa (acción gubernamental) implica que todos los actores dentro del proceso de implementación apunten en la misma dirección sus esfuerzos. (Martínez, 2010, p.18).

Otros estudios, como el realizado por Repetto (2010), afirman que el éxito de la gestión pública está relacionado con el enfrentamiento a retos, en términos de relaciones intergubernamentales e intersectoriales. Para el autor, se entiende por coordinación al:

[...] proceso mediante el cual se va generando sinergia entre las acciones y los recursos de los diversos involucrados en un campo concreto de la gestión pública, al mismo tiempo que en dicho proceso se van construyendo un sistema de reglas de juego formales e informales, a través del cual los actores encuentran fuertes incentivos para cooperar, más allá de sus intereses o ideologías particulares (Repetto, 2010, p. 4).

Sin embargo, la coordinación intergubernamental enfrenta múltiples retos. A nivel organizativo se puede enfrentar con la complejidad de diversos ámbitos y niveles, excesiva diferenciación estructural, así como una sectorialización inadecuada. Así también, pueden existir fracturas y segmentaciones organizacionales, presencia de nuevos actores, predominancia de modelos de gestión de baja interdependencia e interacción, entre otros.

Esta identificación de problemas de coordinación ha llevado a abordar la concepción de la coordinación no como un tema operacional, sino como un problema de acción colectiva (Peters, 2013). Es decir, que no basta un nivel 
jerárquico de poder, modelos organizacionales o arreglos formales para lograr que exista una coordinación «eficiente». El autor también hace una diferencia entre coordinación de recursos, entendida como asignación desde el gobierno central; y coordinación de actividades, propia del gobierno subnacional.

Debido a las múltiples perspectivas desde donde se analiza el fenómeno de la coordinación de las políticas públicas, es posible concebirlo como un proceso de múltiples dimensiones (político y técnico, participativo y concertado, vertical y horizontal), y de diferentes alcances (macro, meso y micro), que involucra a diferentes actores y sectores, con el fin de articular esfuerzos y crear sinergias que favorecen el logro de objetivos estratégicos (Molina y Licha, 2005).

Por ello, a fin de delimitar el concepto, en la presente investigación se entenderá por coordinación como un proceso político y técnico que se orienta a la coherencia y articulación, tanto horizontal como vertical, de las políticas públicas a fin de favorecer el logro de objetivos estratégicos (Garnier, 2000). Lo político implica coordinar para enfrentar las contradicciones, restricciones o resistencias de los actores, tomando caminos de cooperación o negociación; mientras que lo técnico se refiere a una articulación operativa que obtenga los instrumentos y dispositivos adecuados para la implementación de políticas, programas o proyectos determinados (Repetto y Fernández, 2012).

\section{VAIVENES EN LA LUCHA CONTRA La CORRUPCión (2000-2015)}

En el Perú, la política anticorrupción se intensificó a partir del año 2000. En 2001, la coyuntura nacional impulsó la implementación del Programa Nacional Anticorrupción y paralelamente se constituyó el Grupo de Trabajo «Iniciativa Nacional Anticorrupción», creado con el objetivo de ejecutar un conjunto de acciones para el diagnóstico y diseño de políticas de lucha contra la corrupción. Esta iniciativa creó un nuevo modelo para generar espacio en el que diferentes actores, tanto públicos como privados, puedan discutir asuntos relacionados con las bases éticas y democráticas que serían las bases del Estado - las cuales fueron muy cuestionadas en la década precedente-y se encamine un proceso de construcción de un marco normativo e institucional anticorrupción (resolución suprema 160-2001-JUS).

En 2002, la creación de la Comisión Nacional de Lucha contra la Corrupción y Promoción de la Ética y Transparencia en la Gestión Pública se convirtió en otro hito en el desarrollo de una política anticorrupción en el país, la cual significó un intento de institucionalizar la lucha contra la corrupción y 
establecer acciones específicas para su ejecución. Esta comisión se creó a inicios del gobierno de Alejandro Toledo y estuvo conformada por las principales entidades del país, como la Presidencia del Consejo de Ministros, el Ministerio de Justicia, la Contraloría General de la República, la Defensoría del Pueblo, entre otros. Esta comisión formalmente estuvo activa hasta 2006, sin embargo, en la práctica no poseía un funcionamiento debido a la falta de un funcionario que ejerciera de presidente (Silva, 2012).

Posteriormente, en 2007, se creó la Oficina Nacional Anticorrupción, adscrita a la Presidencia del Consejo de Ministros (decreto supremo 085-2007PCM). A través de esta oficina se inició la preparación del primer plan nacional de lucha contra la corrupción, el mismo que tendría un periodo de aplicación desde 2008 a 2011 y contendría medidas para prevenir, investigar y promover una ética pública, la vigilancia ciudadana y erradicar la corrupción.

Paralelamente a la aplicación del plan anticorrupción 2008-2011, el año 2007, mediante el decreto supremo 027-2007-PCM, la Presidencia del Consejo de Ministros establece las políticas de obligatorio cumplimiento para todas las entidades del Gobierno nacional. Este dispositivo consiste en requerir a las instituciones, el planteamiento de metas semestrales objetivas y verificables, con el objetivo de impulsar de manera transversal su promoción y ejecución, en adición a las políticas sectoriales. Así, dentro de las políticas nacionales, la política nacional en materia de lucha contra la corrupción (Política Nacional 11) tiene como propósito definir objetivos prioritarios a fin de asegurar una adecuada gestión pública en esta materia, estableciendo una eficiente lucha contra la corrupción y fomentando la transparencia y ética pública.

Luego que la Oficina Nacional Anticorrupción quedara desactivada en 2008, en 2010 se propuso la creación de la Comisión de Alto Nivel Anticorrupción (decreto supremo 016-2010-PCM), en funcionamiento hasta la actualidad, la cual significó un espacio de articulación y coordinación de acciones y políticas de corto, mediano y largo plazo en materia de prevención y combate de la corrupción en el Perú. La CAN Anticorrupción posee actualmente funciones de planificación, coordinación y seguimiento de la política anticorrupción en el país.

Pues bien, a pesar de que el tema anticorrupción estuvo en la agenda pública en los distintos gobiernos, existen claras dificultades que han estado presentes en todo este periodo en ámbito estructural, institucional y operativo. Primero, la acción gubernamental en esta materia presentó serias dificultades a fin de implementar políticas de lucha contra la corrupción constantes y eficientes. Por ejemplo, en el caso de la Oficina Nacional Anticorrupción, hubo muchos 
cuestionamientos alrededor de sus funciones, ya que algunas de ellas, como la investigación de casos de corrupción, colisionaban con las funciones de otros organismos constitucionalmente autónomos, como el Ministerio Público y el Poder Judicial, por ello solo tuvo un periodo de nueve meses de funcionamiento.

Segundo, las acciones implementadas que conformaron la política tuvieron inconvenientes dada la complejidad del fenómeno de corrupción y la articulación del mismo desde la gestión pública (Silva, 2012). La mayoría de iniciativas estuvieron coordinadas e implementadas por el Poder Ejecutivo, excluyendo a los demás poderes y organismos del Estado, lo cual generaba falta de credibilidad en las acciones llevadas a cabo.

Tercero, las iniciativas anticorrupción no contaron en su mayoría con una estrategia o una herramienta de planificación que guiara las acciones a realizar. Según la Convención de las Naciones Unidad contra la Corrupción, se menciona que para una efectiva lucha contra la corrupción los Estados deberán formular, aplicar y mantener en vigor políticas coordinadas y eficaces contra la corrupción a fin de fomentar el respeto al Estado de derecho, la integridad, transparencia y lucha contra la corrupción (ONU, 2003). En el caso del Perú y en países de América Latina, las iniciativas anticorrupción se caracterizaron por ser desarticuladas, sin mecanismos de seguimiento establecidos, significando acciones momentáneas generalmente motivadas por entornos de crisis y escándalos de gran corrupción, haciendo un llamado a las autoridades políticas sobre la gravedad del problema y su efecto sobre la gobernabilidad democrática (Peñailillo, 2011). La planificación y seguimiento son necesarios en la implementación de políticas anticorrupción, de tal manera que orienten a los actores públicos y privados en su implementación, generen información útil para su seguimiento y permitan una actuación más coordinada de los órganos estatales del control político, financiero y administrativo.

\section{Problemas en torno al diseño institucional de la política ANTICORRUPCIÓN EN SUNAT}

La Sunat, al ser la entidad rectora de la recaudación de impuestos y administración de aduanas del país, es vulnerable a la consolidación de redes de corrupción dentro de sus procesos cotidianos de regulación y control. De esta forma, existen informes que analizan el impacto de la corrupción, por ejemplo, en el control aduanero en límites fronterizos, facilitando la incidencia de delitos aduaneros, como el contrabando, la defraudación de rentas o el tráfico de mercancías restringidas (Bardales, 2010). En la actualidad, la Sunat está compuesta 
por 531 unidades operativas a nivel nacional y con más de 12 mil funcionarios públicos en diversas partes del país, lo cual convierte la labor de control gubernamental en un aspecto clave de la gestión.

Se puede afirmar que desde el año 2000 se iniciaron una serie de medidas que buscaron controlar la influencia política dentro de la Sunat y eliminar las rentas de la corrupción consolidadas en la década fujimorista (Durand, 2012). De esta manera, se inició un proceso de institucionalización y refuerzo de la capacidad recaudadora de la entidad, así como procesos de evaluación y auditorías técnicas. De acuerdo a la normativa vigente, dentro de una entidad pública existen dos órganos específicos que intervienen directamente en la prevención, control y sanción de los hechos de corrupción: el Órgano de Control Institucional (OCI) y la Procuraduría Pública.

En primer lugar, se encuentra el Órgano de Control Institucional (OCI), el cual forma parte del Sistema Nacional de Control ${ }^{1}$, cuyo ente rector es la Contraloría General de la República (según el artículo 82 de la Constitución Política). El OCI es el órgano encargado de ejecutar el Control Gubernamental dentro de toda entidad estatal, sea de carácter nacional, sectorial, regional o local, de acuerdo al artículo 3 de la ley 27785 (ley Orgánica del Sistema Nacional de Control y de la Contraloría General de la República), y las disposiciones normativas dadas por la Contraloría. Según especifica en la citada norma:

El OCI constituye la unidad especializada responsable de llevar a cabo el control gubernamental en la entidad, de conformidad con lo dispuesto en los artículos $6^{\circ}, 7^{\circ}$ y $8^{\circ}$ de la ley, con la finalidad de promover la correcta y transparente gestión de los recursos y bienes de la entidad, cautelando la legalidad y eficiencia de sus actos y operaciones, así como el logro de sus resultados, mediante la ejecución de labores de control (ley 27785, artículo 3).

El control interno comprende la realización de acciones de supervisión con la finalidad que la gestión de recursos, bienes y operaciones se realice de forma eficiente. De acuerdo a la normativa, existen tres tipos de control que se pueden implementar dentro de la entidad: control preventivo, simultáneo y posterior. Dentro de las funciones asignadas al OCI se encuentra desarrollar acciones de control simultáneo, las cuales implican presenciar las actividades que realice la entidad, tales como un proceso de contratación o licitación; por otro lado, acciones de control posterior, las cuales son aquellas que se realizan luego de realizada la actividad.

\footnotetext{
1 De acuerdo a la ley 27785, Ley Orgánica del Sistema Nacional de Control y de la Contraloría General de la República.
} 
Las acciones de control llevadas a cabo por el OCI están enmarcadas en el Plan Nacional de Control y en los dispositivos aprobados para cada entidad de acuerdo a su programación de actividades, como el Plan Operativo Institucional (POI). Entre las principales modalidades que realiza el $\mathrm{OCI}^{2}$ se encuentran: por un lado, las visitas de control, que implica la supervisión por parte de un auditor a fin de asegurar que la normativa interna de la entidad se cumpla adecuadamente; por otro lado, las auditorías de cumplimiento, que es la revisión posterior para asegurar el cumplimiento de todos los procedimientos (por ejemplo, durante los procesos de contratación o adquisición).

Luego de la acción de control realizada, si se determinan responsabilidades administrativas ${ }^{3}$, la Contraloría General de la República deberá adoptar acciones para la determinación de responsabilidad y la imposición de la sanción respectiva, según las facultades otorgadas por ley ${ }^{4}$. Por otro lado, si se determinan indicios razonables de responsabilidad civil ${ }^{5}$ o penal $^{6}$, el jefe del Órgano de Auditoría Interna comunicará sobre las responsabilidades simultáneamente al procurador público para que este realice las funciones competentes.

Sobre este último punto, se puede identificar a la Procuraduría Pública como el segundo actor protagonista del control gubernamental en una entidad pública. La procuraduría es el órgano encargado de la representación y defensa jurídica de los derechos e intereses del Estado. Al igual que el caso de los órganos de control institucional, depende administrativamente de la entidad, en este caso Sunat, y, funcionalmente, del Consejo de Defensa Jurídica del Estado (Ministerio de Justicia y Derechos Humanos). Según el artículo 27 del Reglamento de Organización y Funciones de Sunat, se especifica como función principal de la Procuraduría:

Representar al Estado y defender los derechos e intereses de la Sunat ante los órganos jurisdiccionales y administrativos, así como ante el Tribunal Constitucional, el Ministerio Público, la Policía Nacional, el Tribunal Arbitral, el Centro de Conciliación y otros de similar naturaleza en los que el Estado es parte, comprendiendo todas las actuaciones en materia procesal, arbitral y de carácter sustantivo que permite la ley.

\footnotetext{
2 De acuerdo a la resolución de contraloría 273-2014-CG «Normas Generales de Control Gubernamental».

3 Es aquella en la incurren los servidores y funcionarios por haber contravenido el ordenamiento jurídico administrativo y las normas internas de la entidad a la que pertenecen, se encuentre vigente o extendido el vínculo laboral, durante el desarrollo de la acción de control. Así también aquellos funcionarios que desarrollaron una gestión ineficiente.

4 La Contraloría General de la República y el Tribunal Superior de Responsabilidades Administrativas ejercen la potestad sancionadora conferida por la ley 29622, ley que modificó la ley 27785, Ley Orgánica del Sistema Nacional de Control y de la Contraloría.

5 Aquellos funcionarios que por su acción u omisión en el ejercicio de sus funciones hayan ocasionado un daño económico a su entidad o al Estado, incumpliendo sus funciones.

6 Aquella en la que incurren los servicios o funcionarios que en ejercicio de sus funciones han efectuado un acto $\mathrm{u}$ omisión tipificado como delito.
} 
Como se mencionó anteriormente, si se ha determinado responsabilidades penales, la oficina encargada de viabilizar y encaminar dicho caso es la Procuraduría Pública. Esta oficina realiza una investigación sobre el caso reportado por el OCI (generalmente), y si se encuentran evidencias, se presenta formalmente el expediente al Ministerio Público ${ }^{7}$. Luego, en dicha institución se realiza una investigación para formalizar el caso ante el Poder Judicial, de acuerdo al proceso de investigación penal de la corrupción.

Figura 1. Modelo de investigación de actos de corrupción OCI y Procuraduría

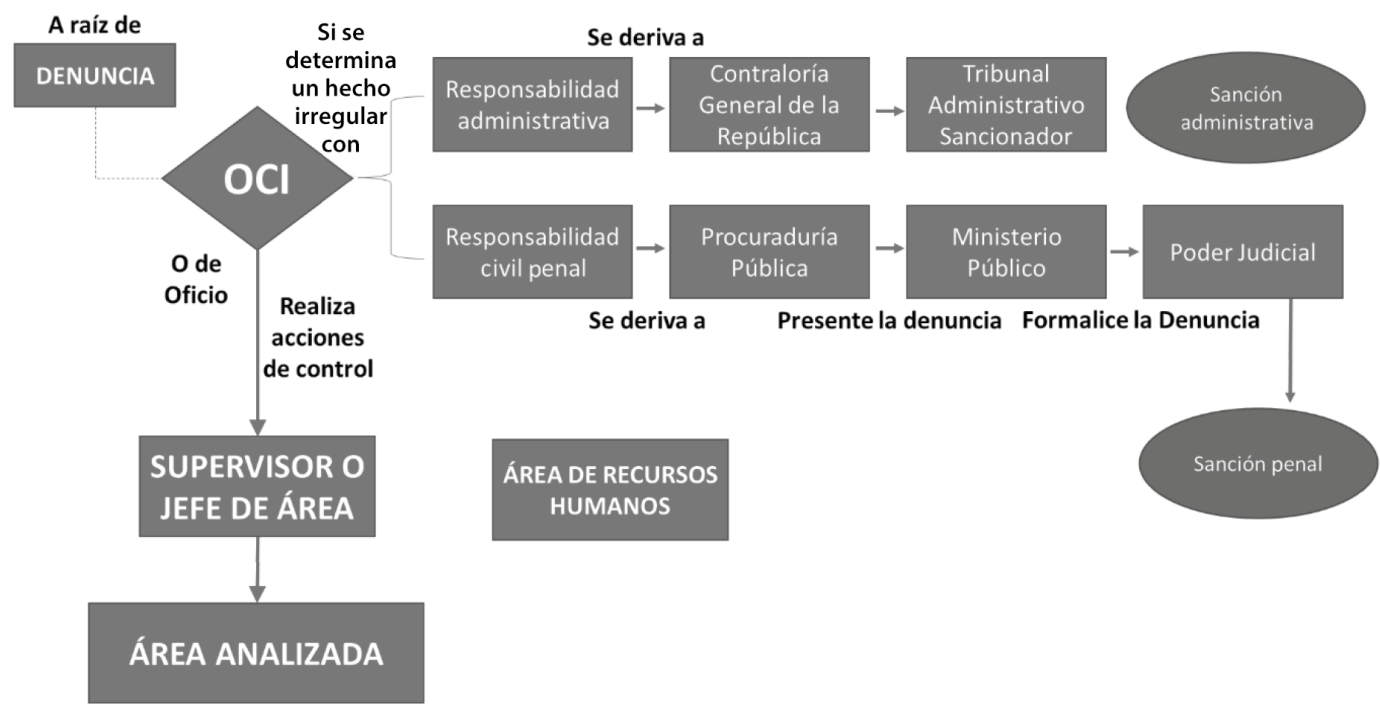

Fuente y elaboración propia.

De acuerdo a lo mencionado anteriormente, el marco normativo del control gubernamental establece al Órgano de Control Institucional como principal actor de la lucha contra la corrupción al interior de la entidad. Sin embargo, de acuerdo a las entrevistas realizadas durante el trabajo de campo, se ha podido identificar ciertos problemas operativos.

Primero, el tiempo de duración del proceso. Como se indicó, el proceso inicia con la investigación, sea de oficio u originado por una denuncia, realizada por el OCI. Sin embargo, hasta que dicha investigación pase por las todas

\footnotetext{
7 Según el Nuevo Código Procesal Penal, el Ministerio Público se encuentra encargado de la investigación preparatoria para los delitos contra la administración pública, de acuerdo a la ley 29574.
} 
las fases del proceso, es posible que no llegue a una sanción efectiva del acto de corrupción identificado. En esta línea, se ha recogido el siguiente testimonio:

Los informes que elaboran los OCI están sujetos al control de calidad, de la Contraloría General de la República, la cual tiene la facultad de aprobar o disponer su reformulación. Desde hace unos años, la Contraloría adopta una función sancionadora, por lo que antes de pasarle el expediente al procurador la Contraloría tiene que dar el visto bueno. Se ha tenido casos donde se determina responsabilidad administrativa y no se sanciona, pues al haberse demorado el proceso seis o siete meses, el trabajador puede apelar al principio de inmediatez (Funcionario 1 Oferlccor, 9 de junio 2016).

Segundo, en general, las funciones de supervisión del OCI se restringen a verificar que los procedimientos de compras y adquisiciones públicas se desarrollen de acuerdo a ley, no profundizando en la fiscalización en las labores cotidianas de la entidad. Es decir, aquellas que implican contacto entre los funcionarios públicos y la ciudadanía y en las que se pueden presentar prácticas que no necesariamente están tipificadas como delitos de corrupción (ámbito penal) o faltas administrativas (ámbito administrativo).

Tercero, existe el riesgo de que el proceso se frustre. Durante la investigación judicial en el Ministerio Público — y de ser el caso en el Poder Judicial—, debido al amplio periodo de duración del proceso, existen potenciales riesgos de que no se llegue a una sanción efectiva.

Cuando no solo se determina responsabilidad administrativa sino también se determina responsabilidad penal actúa el Ministerio Público. Ahí viene otro problema... En la práctica la formalización de una denuncia ante el Poder Judicial se demora dos o tres ańos. Además, existe la posibilidad que el fiscal sea corrupto, mal preparado, o simplemente ineficiente y que las gestiones realizadas hasta el momento simplemente no lleguen a nada. Si se llega a la etapa del Poder Judicial puede tomar tres o cuatro años en dictar sentencia de encontrar hallazgos suficientes. Nuevamente, se puede dar el caso en que exista un juez que haga que el caso y todo el procedimiento logrado no otorguen una resolución (Funcionario 2 Oferlccor, 1 de julio del 2016).

Sobre este punto, hay evidencias de funcionarios de la institución que, tras haber sido denunciados por corrupción y separados de la institución, posteriormente fueron restituidos por medidas cautelares y resoluciones del Poder Judicial (El Comercio, 2009). Esto debido a que durante el proceso no se llevó a cabo una adecuada presentación de la evidencia ni ciertas características formales del proceso de investigación penal que terminaron en un fallo cuestionable del Poder Judicial. 


\section{La Oficina de Fortalecimiento Ético y Lucha contra la Corrupción: MECANISMOS DE COORDINACIÓN Y PRINCIPALES LIMITACIONES}

En la Sunat, a partir de 2011 se encaminaron reformas a fin de implementar adecuadamente el Plan Nacional de Lucha contra la Corrupción emitido por el Poder Ejecutivo. De esta manera, en el año 2012 se creó la Oficina de Fortalecimiento Ético y Lucha contra la Corrupción (Oferlccor), cuyo objetivo fue fortalecer e institucionalizar la política anticorrupción en la entidad. Actualmente la oficina, según el organigrama de la Sunat, depende directamente de la Superintendencia, al igual que otras áreas, como la Secretaría General, y cuenta con trece funcionarios designados.

Para comprender la evaluación de las medidas anticorrupción en Sunat y el funcionamiento de dicha área es necesario mencionar que su funcionamiento no inició netamente con el plan, sino que se articuló con otro conjunto de actividades que realizaba el Órgano de Control Institucional de Sunat. Lo mencionado se puede explicar en el siguiente testimonio:

Nuestra llegada al plan fue a la inversa. Nosotros analizábamos casos que se detectaban en la Oficina de Control Interno, los casos que estaban judicializados y de ahí partíamos con análisis de casos para determinar aquellas situaciones que facilitaban la incidencia de casos de corrupción [...] (exfuncionario Sunat, 18 de mayo del 2016).

A partir del ańo 2012, la Oferlccor ha tenido como una de sus principales funciones la implementación del Plan Sectorial formulado por el MEF, y a su vez la formulación del Plan Institucional de Sunat, el cual comprende una serie de actividades y acciones a ejecutar durante un periodo anual.

El plan institucional anticorrupción de Sunat, si bien ha variado desde su emisión en el año 2011, se divide en tres grandes tipos de acciones según su objetivo. Primero, los que están dirigidos a realizar una sensibilización sobre temas vinculados a ética en la función pública en la institución, en el cual figuran actividades como realizar eventos de difusión sobre temas de ética, realizar encuestas externas e internas sobre percepción de la corrupción, y realizar campañas internas para difundir los logros y avances en la implementación del plan institucional.

Segundo, las actividades relacionadas con prevención de la corrupción. Entre las más importantes figuran la ejecución de visitas inopinadas a las diversas áreas de la entidad, y que previamente han sido identificadas como áreas vulnerables a riesgos de corrupción; y realizar evaluaciones periódicas sobre este tipo de diagnósticos. Mediante las visitas se plantean recomendaciones a la Alta 
Dirección sobre situaciones que estén relacionadas con el incumplimiento de la normativa interna, previniendo potenciales hechos de corrupción.

Tercero, acciones internas de lucha contra la corrupción. En este ámbito cabe resaltar dos actividades que realiza la Oferlccor. Por un lado, las actividades de identificación de áreas y procesos con riesgos de corrupción dentro de Sunat, mediante la revisión de los casos que han sido detectados con anterioridad. Por otro lado, la realización de asesorías en las labores de control que le corresponde a los funcionarios o jefes de las distintas áreas de Sunat.

\section{Tipología de mecanismos de coordinación}

La coordinación gubernamental comprende un constructo organizacional, la cual debe sostenerse a través de una serie de instrumentos concretos (Repetto y Fernández, 2012). La implementación de la estrategia nacional anticorrupción en planes de acción requiere procesos de coordinación y seguimiento a fin de alcanzar la realización de los objetivos y resultados efectivos (Hussmann, 2007).

En el caso de la política anticorrupción, no se ha profundizado el estudio sobre su implementación ni las dinámicas que implican dentro de las instituciones públicas. En el Poder Ejecutivo se han desarrollado un conjunto de mecanismos a fin de optimizar la coordinación interinstitucional de los actores. Según el caso de estudio elegido, la Oficina de Fortalecimiento Ético y Lucha contra la Corrupción en Sunat, se puede organizar los distintos mecanismos según los alcances posibles.

\section{i. Mecanismos de coordinación para la investigación de actos de corrupción}

Si bien cuando se creó la Oferlccor, esta asumió la función de formular herramientas de prevención de la corrupción dentro de la institución, a inicios de 2015, mediante la resolución de Superintendencia 400-2014/Sunat, la Oferlccor adquirió las funciones para brindar asesoría y apoyo a los funcionarios o directivos de la institución en el ejercicio del control y lucha contra la corrupción. Es decir, la oficina que inicialmente tenía las funciones íntegramente preventivas, comenzó a participar y asesorar en procesos de investigación de presuntos actos de corrupción.

Cabe mencionar que uno de los obstáculos que atravesó el área durante la adopción de la función de investigación fueron las constantes críticas de duplicación de funciones por parte del OCI. 
Figura 2. Funciones de asesoría y apoyo en el ejercicio del control a directivos

\section{Asesoría}

- Consiste en la orientación o sugerencias que brinda la Ofelccor a un funcionario o directivo de la Sunat con relación a las consultas formuladas de manera verbal o escrita respecto a la forma de cumplir con las labores que le son inherentes vinculadas al ejercicio del control que le corresponde.

\section{Apoyo}

- Consiste en la labor de evaluación y de programación de la investigación (de corresponder) que realiza la Ofelccor al recibir una solicitud de parte de un funcionario o directivo de la Sunat para ejecutar labores que le son inherentes vinculadas al ejercicio del control. El apoyo consiste en realizar una investigación, emitiendo como resultado un informe técnico.

Fuente: Oferlccor. Elaboración propia

Figura 3. Modelo de asesoría y apoyo en el ejercicio del control

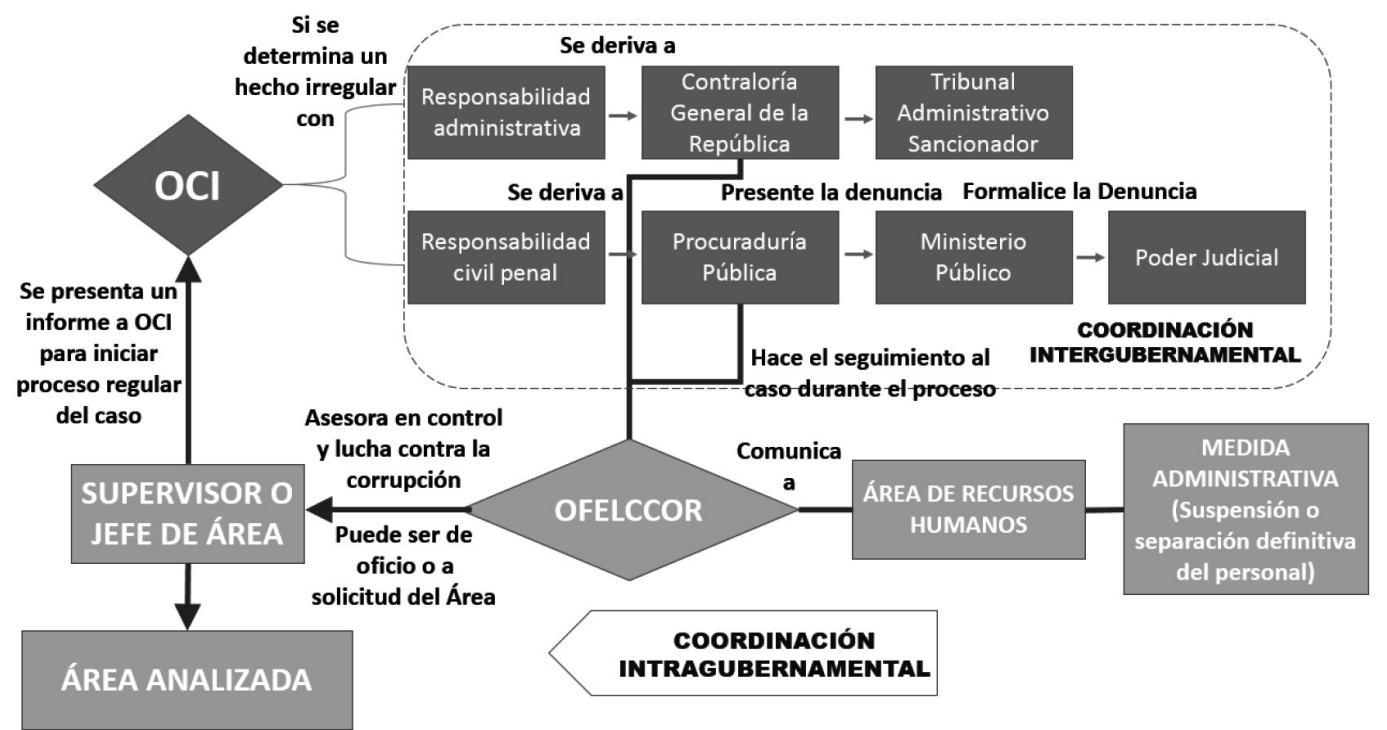

Fuente: Oferlccor. Elaboración propia. 
Sin embargo, el mecanismo propuesto por la Oferlccor plantea que esta no es la encargada directa de realizar la investigación —como sí lo es el Órgano de Control Institucional—, sino que es la encargada de apoyar y asesorar a los directivos, de acuerdo a la función brindada por la ley de Control Interno, la cual especifica lo siguiente:

El control interno posterior es ejercido por los responsables superiores del servidor o funcionario ejecutor, en función del cumplimiento de las disposiciones establecidas, así como por el Órgano de Control Institucional según sus planes y programas anuales, evaluando y verificando los aspectos administrativos del uso de los recursos y bienes del Estado, así como la gestión y ejecución llevadas a cabo, en relación con las metas trazadas y resultados obtenidos (ley 27785, artículo 7).

De esta manera, no existe una contraposición entre las facultades del OCI y la Oferlccor, siendo el primero el receptor de las denuncias de la ciudadanía y la implementación del control interno de acuerdo a las normas emitidas por la Contraloría; y el segundo, un órgano de asesoría en investigaciones de presuntos actos de corrupción.

El Órgano de Control Institucional trata las denuncias que ingresan por ciudadanos o por terceros. Nosotros vemos las investigaciones que los directivos nos solicitan cuando existen indicios de actos de corrupción dentro de sus áreas respectivas (Funcionario 1 Oferlccor, 9 de junio 2016).

Un punto importante que es necesario mencionar es la coordinación que se realiza entre la Oferlccor y los distintos órganos del proceso penal de investigación de actos de corrupción. Si bien la función de la oficina culmina con la emisión del informe detallando los resultados de la investigación, luego que el caso ingresa en el proceso regular, se realiza seguimiento para evitar que se estanque en alguna fase del mismo.

A pesar que nuestra función es la emisión del informe, hacemos seguimiento de nuestros casos con los fiscales al que han sido derivados, dado que es necesario no descuidar el proceso. Así también se coordina con la Procuraduría Anticorrupción para que el caso no se congele en alguna entidad dentro del proceso o se pierda dentro de todos los casos que tienen (Funcionario 1 Oferlccor, 9 de junio 2016).

Según las estadísticas de la Oferlccor, desde la aprobación de las facultades de investigación a inicios de 2015 hasta mediados de 2016, se han llevado a cabo 35 casos de investigación por actos de corrupción, de los cuales en 17 se han determinado responsabilidades por funcionarios de la institución. Respecto a la duración de la investigación, en promedio la oficina culmina un caso en 30 días, mientras que en el OCI puede llevar hasta un año de duración. 


\section{ii. Procedimientos de detección de riesgos de corrupción}

La corrupción es un complejo objeto de medición, generando que la tarea de evaluar o medir la corrupción sea particularmente desafiante. La medición de la corrupción se ha convertido en un tema central para el estudio de la buena gobernanza y una demanda para el diseño e implementación de políticas públicas de lucha contra la corrupción, la cual ha aumentado su presencia en la agenda pública a nivel internacional en las últimas décadas (Parker, Berthin, Michelle y Mizrahi, 2004).

Un delito como la corrupción difícilmente puede ser registrado en un estudio de victimización pues los partícipes de estos actos conocen la situación no necesariamente como víctimas, sino como cómplices, lo que dificulta la medición (Mujica, 2013, p. 176).

Dentro de las políticas anticorrupción llevadas a cabo por las entidades del Estado, el poseer un diagnóstico ha sido una de las tareas más complejas para asegurar una correcta implementación de la política en cada entidad. De acuerdo a lo mencionado por un asesor de la CAN Anticorrupción:

Cuando revisábamos los planes anticorrupción, tanto a nivel sectorial como los planes de las comisiones regionales anticorrupción, descubrimos que el diagnóstico muchas veces es malentendido por muchos servidores públicos, reduciéndolos a una recopilación normativa de la entidad o con indicadores de ámbito nacional o a un nivel muy general (asesor CAN, 1 de octubre 2016).

La Oferlccor, en el año 2014, desarrolló una metodología propia para llevar a cabo un diagnóstico institucional para poder identificar las áreas que presentan mayor riesgo de posibles ocurrencias de corrupción (Oferlccor, 2014). De acuerdo a su metodología, ellos parten de entender la corrupción como:

Toda conducta que realice un funcionario o servidor público que implique el incumplimiento de funciones o el quebrantamiento de la legalidad, con el objeto de obtener un beneficio extraposicional indebido, propio o para terceros; sea de carácter económico, administrativo o de cualquier otra índole (Oferlccor, 2014).

En dicho proceso se establecieron cuatro áreas a evaluar, que determinarían el nivel de riesgo que tendría un área a la incidencia de actos de corrupción. Las áreas evaluadas son: discrecionalidad del funcionario público para la toma de decisiones, frecuencia de contacto con el público, nivel de informatización de los procesos del área y el grado de supervisión en el que se encuentra.

Empezamos a analizar en base a la casuística, cómo medir el riesgo de actos de corrupción. [...] Cuando concluyen los cuatro o tres de estos factores podemos determinar un área de alto riesgo (Funcionario 2 Oferlccor, 1 de julio 2016). 
Si bien inicialmente existió la limitación de que la fuente principal de información eran autoevaluaciones de las mismas áreas (o unidades operativas) de acuerdo a su propia experiencia, posteriormente se cruzó la información recopilada con los resultados de investigaciones anteriores, tanto las realizadas por la Oferlccor como por el Órgano de Control Institucional, lo que permitió tener un análisis más fino de las áreas más vulnerables.

Este diagnóstico serviría como instrumento de gestión, que guiaría la implementación de estrategias de prevención e investigación dentro de la entidad y sobre todo la realización de visitas inopinadas como mecanismo de supervisión.

Las áreas son evaluadas por cada factor para llegar a una tabulación y aquellas que tienen altos puntajes son aquellas que nosotros priorizados por el alto riesgo. Con la metodología se pudo determinar un número reducido de áreas (34). Con ello se pudo determinar y dirigir las visitas, las investigaciones, los mecanismos de reducción de riesgos hacia estas áreas identificadas (Funcionario 2 Oferlccor, 1 de julio 2016).

La evaluación se realizó en las 531 unidades operativas ${ }^{8}$ (áreas) que componen la estructura de la Sunat, con lo cual se construyó un mapa de riesgos de corrupción, en un periodo de dos meses aproximadamente. Entre los principales resultados de la utilización de esta metodología está la determinación de prioridades para la realización de investigaciones.

Finalmente, cabe mencionar que, sin un adecuado diagnóstico de la problemática particular en cada entidad, una iniciativa anticorrupción sin vinculación a los problemas y necesidades reales de la institución puede devenir en solo un «incremento de la burocracia o en soluciones sin sustento real» (Peńailillo, 2011).

\section{iii. Creación de la Oficina Anticorrupción}

Una diferencia que se ha podido evidenciar durante la implementación del Plan Nacional de Lucha contra la Corrupción 2012-2016, es la creación de oficinas anticorrupción al interior de las entidades del Estado. Es decir, además de la Comisión de Alto Nivel Anticorrupción, adscrita a la PCM —la cual tiene la función de hacer seguimiento al plan nacional anticorrupción-, en algunas entidades se han implementado oficinas específicamente encargadas de la prevención y lucha contra la corrupción.

\footnotetext{
8 Las unidades operativas bajo estudio fueron: 18 de la Superintendencia Nacional, 56 de la Superintendencia Nacional Adjunta de Desarrollo Estratégico, 224 de la Superintendencia Nacional Adjunta Operativa, 147 de la Superintendencia Nacional Adjunta de Aduanas y 86 de la Superintendencia Nacional Adjunta de Administración y Finanzas.
} 
En el ámbito del Poder Ejecutivo, si bien en la mayoría de casos las entidades optan por encargar a la Oficina de Planeamiento y Presupuesto la labor de seguimiento del plan institucional anticorrupción, ciertas entidades han creado un área específicamente encargada de formular, implementar y monitorear la política anticorrupción en su jurisdicción. En el caso de estudio, dentro de la Sunat, la Oferlccor, al aplicar el enfoque de prevención de forma simultánea con el enfoque combativo, marca una práctica relevante dentro de la lucha contra la corrupción en el país.

\section{iv. Espacios de coordinación intersectorial}

Desde la Presidencia del Consejo de Ministros, la Comisión de Alto Nivel Anticorrupción coordinó tanto con el MEF como con los distintos órganos y empresas adscritos al sector, a fin de desarrollar adecuadamente las estrategias institucionales anticorrupción.

En julio de 2013, la CAN Anticorrupción realizó el primer «Taller de intercambios de experiencias» a fin de compartir información con todos los ministerios. Así también fue una oportunidad para que los funcionarios de cada sector pudieran comentar sobre las buenas prácticas y los problemas operativos durante la formulación e implementación de los planes anticorrupción.

Según lo mencionado por un asesor de la Oferlccor, las reuniones de coordinación han permitido intercambiar información importante sobre la lucha anticorrupción en otras entidades para aplicarlo en Sunat:

Las reuniones de coordinación interinstitucional son fundamentales porque recogen las experiencias de otras entidades y explican qué es lo que se ha hecho. Luego de las conversaciones se pueden extraer buenas prácticas que pueden implementarse en nuestra entidad (Funcionario 1 Oferlccor, 9 de junio 2016).

En la misma línea, la coordinación interinstitucional también ha impactado en el desarrollo de la capacidad organizativa de las entidades a través de la designación de los coordinadores de enlace ministerial. En marzo del año 2013 la CAN Anticorrupción solicitó formalmente a los ministerios la designación de un personal como "coordinador de enlace» a fin de mantener comunicación y coordinar las acciones para la implementación del Plan Nacional de Lucha contra la Corrupción 2012-2016.

\section{v. Sistemas de información compartida}

Dentro de la implementación de la política anticorrupción en la Sunat, la informatización de los procesos tuvo un papel central. 
A partir del año 2014, la Sunat incorporó en su plan sectorial anticorrupción la implementación del Registro de Procedimientos Administrativos vinculados a la corrupción (Reprac) como instrumento de gestión de información. El Reprac es un instrumento de recolección de información en el cual todas las entidades reportan todos los procesos y procedimientos disciplinarios que se han aplicado a su personal, referentes a temas vinculados a corrupción.

El equipo técnico de la CAN realizó capacitaciones al personal del área de Planeamiento y Presupuesto de la Sunat para el uso de la aplicación. Los reportes se realizan de forma trimestral a la CAN Anticorrupción y tienen como principal objetivo obtener información estadística rigurosa que facilite la toma de decisiones para la mejora o corrección de la conducta del funcionario público (directiva 002-2012-PCM/SGP). Según el coordinador adjunto de la CAN Anticorrupción:

A diferencia de otros, en el Ministerio de Economía tenían un equipo técnico que poseían mayor conocimiento en temas de planeamiento estratégico y formulación de indicadores, por lo que el proceso se pudo realizar de una forma más ágil.

Posteriormente, dicha iniciativa fue simulada en otras instituciones y ministerios dentro de sus planes sectoriales anticorrupción.

\section{vi. Normativa e instrumentos de planificación}

Según los funcionarios de la CAN, desde la Presidencia del Consejo de Ministros, con la emisión de la Estrategia Anticorrupción del Poder Ejecutivo, si bien la mención de los planes institucionales ya aparecía en el plan, con este dispositivo legal se podría hacer más viable la influencia desde la PCM hacia los distintos sectores a fin de promover la emisión de planes anticorrupción.

[La CAN Anticorrupción] fue influyente desde que emitieron la política nacional, porque fue una herramienta legal que obligó a todas las entidades del sector público a poder alinearse a esa política. Si no hubiesen sacado la ley probablemente ni siquiera hubiésemos pensado en diseñar estas herramientas (Funcionario 1 Oferlccor, 9 de junio 2016).

En otras palabras, se realizaba una incidencia dentro de los procesos de toma de decisiones del ministerio y la generación de voluntad política. De acuerdo con un estudio realizado por el U4 Anticorruption Centre, se sostiene que:

Donde la coordinación sigue siendo un reto, comités u organismos especializados pueden ser establecidos para abordar la cooperación entre agencias. Pueden estar compuestos por representantes de los poderes ejecutivo, judicial, legislativo y funcionarios de los departamentos clave (como las costumbres, las adquisiciones, la recaudación de impuestos y cumplimiento de la ley y de los gobiernos locales). También pueden incluir 
a miembros de la sociedad civil, tales como representantes de empresas, organizaciones no gubernamentales, líderes religiosos, etc. En todos los casos, tienen que tener el poder suficiente, los recursos y la capacidad para hacer frente a los ministerios poderosos (Chêne, 2009, p. 7).

En este sentido, el papel de la PCM, como cabeza de los ministerios, y específicamente de la CAN Anticorrupción, como ente rector de la política anticorrupción en el país, fue importante en el proceso de aplicación del plan anticorrupción a nivel sectorial. Es decir, se puede identificar una relación de coordinación vertical mediante la incidencia política a través de reuniones entre la Coordinación General de la CAN y el ministro de cada sector, a fin de ejercer presión para la implementación de los planes institucionales.

Así también, desde una perspectiva intrainstitucional, el contar con el respaldo del marco normativo interno (resolución de superintendencia), permite que la Oferlccor pueda tener el soporte legal para la ejecución de las funciones asignadas. Siguiendo lo mencionado por Heilbrunn (2004), el primer factor que podría explicar la incapacidad de las oficinas anticorrupción es la ausencia de leyes necesarias para que los funcionarios que componen una institución reconozcan las funciones de investigación y lucha contra la corrupción de dicha área.

\section{Limitaciones de los mecanismos de coordinación en la lucha contra la corrupción en Sunat}

De acuerdo a un estudio del PNUD, la construcción de medidas anticorrupción requiere capacidad política, junto con capacidades institucionales y de gestión (PNUD, 2013). Esta investigación, a partir del análisis de caso de la Oferlccor en Sunat, propone que la implementación de una política anticorrupción a nivel de las entidades del Estado presenta limitaciones en cuatro aspectos principales de la política pública: político, técnico, institucional y organizativo-administrativo.

Respecto al aspecto político, según lo recogido en el trabajo de campo, la voluntad política que existe en la élite de la entidad, específicamente centrada en la Superintendencia Nacional y el gabinete de asesores, representa una seria limitación a la política anticorrupción. La coordinación, si bien tiene un alto componente técnico, es una función esencialmente política (Garnier, 2000), la cual tiene como propósito integrar las distintas decisiones y acciones de gobierno dentro de una sola visión de conjunto y con una perspectiva a largo plazo.

En el caso de la Sunat, los entrevistados manifestaron que, si bien existía una corriente que se fue formando desde inicios de los años 2000 y que fue 
madurando al correr de los años, el apoyo de la Superintendencia Nacional podía «acelerar o entorpecer» la consolidación de las políticas anticorrupción. La presión que se presentó en determinados procesos de investigación resultó una seria limitación para las funciones y la autonomía de la Oficina Anticorrupción de Sunat.

En realidad, no es por leyes o normas donde sentimos el apoyo, sino cuando te permiten investigar. De igual manera, sientes que te restan apoyo cuando te quitan herramientas para trabajar (exfuncionario Sunat, 18 de mayo del 2016).

Por ello, una clara limitación identificada en el presente estudio es la subsistencia del enfoque top down en la implementación de políticas públicas anticorrupción. Para el desarrollo de políticas anticorrupción se requiere una coordinación desde el punto de vista político, es decir, relacionamiento de actores que promueva la «agendación» a nivel institucional de las políticas que ya han sido emitidas por instituciones de alto nivel gubernamental. En estos casos, siguiendo a Cortázar (2007), los líderes de las instituciones tienen la capacidad de "problematizar» la corrupción dentro de sus jurisdicciones para el inicio de acciones concretas. En otras palabras, la alta dirección puede agilizar o entorpecer los procesos de implementación de políticas públicas, en especial las referidas a la lucha contra la corrupción.

En cuanto al aspecto técnico, un factor importante es la capacidad burocrática y los conocimientos técnicos que posean los hacedores de política, aquellos que llevan a implementar el contenido de los instrumentos de planificación anticorrupción. Para resolver un problema es preciso identificarlo previamente. En esta línea, existen ineficientes sistemas de medición y monitoreo de riesgos de corrupción para la planificación de acciones operativas. Si bien en la Oferlccor se llevó a cabo un proceso de diagnóstico de riesgo de corrupción, este no se actualizó periódicamente, por lo que podría estar descontextualizado de los cambios que ha sufrido la institución desde entonces. Además, como mencionamos, al estar basado principalmente en encuestas de percepciones, podría estar sesgada dicha información. Por otro lado, también se han puesto en evidencia las desiguales capacidades técnicas en materia de lucha contra la corrupción entre las distintas organizaciones (inclusive entre los fiscales y jueces que analizan los procesos de casos de corrupción).

Con respecto al ámbito institucional, los mecanismos de coordinación están determinados por los procesos internos de la entidad, así como la clara asignación de funciones y competencias. Para Garnier (2000), para que exista una continuidad en las políticas públicas implementadas por una institución, 
aparte del eficaz manejo de recursos humanos y personal técnico, debe estar acompañada de la consolidación institucional, a través de los instrumentos de planificación. De acuerdo con lo propuesto por Repetto:

Los procesos tendientes a promover la coordinación pro-integralidad de las intervenciones estatales en materia de programas y políticas sociales (así como su alcance), están fuertemente condicionados tanto por ciertas características de la institucionalidad formal e informal como por algunos rasgos del entramado de actores que operan de un modo directo o indirecto en la gestión pública de lo social. (2010, p. 27)

En el caso de la Oferlccor, la adopción de facultades de investigación y asesoría en las labores del control interno a los distintos directivos de la entidad representa una buena práctica de coordinación interna. En este escenario, el Órgano de Control Institucional se ve complementado con las acciones de asesoría realizadas por la Oferlccor, pues de encontrar hallazgos relevantes, esta puede presentar el caso para que siga su proceso regular. Así también, dicha coordinación no se limitaba al ámbito intrainstitucional, sino que dicha área puede realizar seguimiento a los casos de investigación que son derivados a la Contraloría o al Ministerio Público, de acuerdo a las responsabilidades detectadas, para asegurar que el proceso siga su curso y llegue a una sanción efectiva del personal que haya incurrido en actos de corrupción. Sin embargo, aun así, se ha evidenciado que las características dentro de la entidad (Sunat), del sector (Economía y Finanzas), así como la ingeniería institucional del sistema de justicia (la duración de los procesos de investigación penal) pueden afectar la implementación de medidas anticorrupción dentro de una entidad. Asimismo, el solapamiento de distintos dispositivos de política anticorrupción, tales como el PNA 2012-2016 y las Políticas de obligatorio cumplimiento (decreto supremo 027/2007-PCM), facilita la ocurrencia de duplicación de funciones, perdiendo de vista la solución del problema público en cuestión.

Finalmente, en cuanto al aspecto organizativo/administrativo, en el caso de estudio, como se mencionó, la Oficina de Fortalecimiento Ético y Lucha contra la corrupción solo cuenta con trece funcionarios, de los cuales solo cuatro poseen la función para realizar los procesos de investigación dentro de las distintas áreas de Sunat.

A comparación de OCI, que cuentan con aproximadamente 100 funcionarios, la oficina posee poco personal que en muchas ocasiones nos limita la cantidad de investigaciones que podemos llevar a cabo. Así también, requerimos recursos para la realización de viajes y visitas inopinadas a unidades que se encuentran dentro del país, lo cual genera un gasto, en especial cuando se presentan solicitudes de asesoría por parte de los directivos de algún área (Funcionario 2 Oferlccor, 1 de julio del 2016). 
De esta manera, un aspecto estratégico en las políticas anticorrupción son las capacidades logísticas de las oficinas encargadas de su implementación. Las entidades que tienen numerosos órganos y dependencias desconcentrados, como el caso de Sunat (entre estos los puestos de control aduanero, las divisiones de control de deuda y cobranza, las divisiones de control fronterizo y coordinación territorial), exigen capacidades de movilización a lo largo del territorio nacional, por lo que es necesario contar con el presupuesto y facilidades logísticas para ello: «[...] las entidades deberán incorporar sus acciones anticorrupción en sus Planes Operativos Institucionales (POI) para asegurar la asignación presupuestal y el recurso humano necesario para su ejecución, así como la realización del seguimiento, monitoreo y evaluación de dichas acciones» (CAN, 2014, p. 5).

Si bien, según la CAN Anticorrupción, las acciones que figuran en el Plan Institucional Anticorrupción deben estar incorporadas en sus Planes Operativos Institucionales, esto en la mayoría de casos no asegura que se brinde el presupuesto requerido, presentándose las limitaciones logísticas y de recursos humanos mencionadas. De acuerdo a lo afirmado por Repetto y Fernández (2012), no es suficiente la existencia de una planificación y articulación con los dispositivos de política nacional y la definición de atribuciones entre las áreas involucradas de una política pública, sino que es imprescindible que sean reafirmadas en los planes y presupuestos de las instituciones estatales.

\section{Conclusiones}

Las políticas anticorrupción se han convertido en uno de los principales aspectos dentro de la problemática de la reforma y modernización del Estado. Recientes estudios han concluido en la necesidad de instaurar políticas integrales anticorrupción (Pautrat, 2016) que puedan articular entre las distintas entidades medidas objetivas de lucha contra la corrupción.

La presente investigación tuvo como objetivo identificar los mecanismos de coordinación de la política anticorrupción en la Sunat y analizar sus principales limitaciones. Estudiar el caso de la Sunat y el funcionamiento de la Oferlccor ha sido muy útil para identificar los alcances que pueden tener las medidas anticorrupción dentro de las entidades del Estado, así como identificar ciertos problema-tipo que podrían ser detectados en otras instituciones públicas (o privadas) donde se desee implementar medidas anticorrupción. 
La política anticorrupción posee un marco normativo de dispositivos de política nacional, tales como el Plan Nacional de Lucha contra la Corrupción o la Estrategia Anticorrupción del Poder Ejecutivo, para brindar lineamientos de acción a las entidades en la lucha contra la corrupción. Sin embargo, es necesario contar con dispositivos adaptados a las características particulares de cada institución pública a fin de que se logre un impacto real en los niveles de corrupción detectados. Por ello, la formulación de Planes Institucionales Anticorrupción es una forma de brindar una mirada más local a la lucha contra la corrupción, así como asegurar una adecuada articulación e implementación de las políticas nacionales.

Las políticas anticorrupción son procesos complejos que requieren la coordinación de numerosos actores en distintos momentos. La coordinación gubernamental es un requisito indispensable en la implementación de la política anticorrupción. De acuerdo al trabajo de campo, el contar con un área encargada de la coordinación e implementación de la política anticorrupción dentro de una entidad es necesario para el adecuado planteamiento de estrategias y acciones operativas internas. Esto pues, si dicha política es llevada a cabo por otras áreas de la entidad (como por ejemplo las Oficinas de Planeamiento y Presupuesto), se corre el riesgo de que la lucha contra la corrupción se convierta en un proceso burocrático formal, entendiendo las tareas o acciones como finalidades últimas y no como medios para resolver un problema particular.

Las estrategias institucionales deben estar basadas en un adecuado diagnóstico de la corrupción, por lo que la coordinación entre las diferentes áreas de una entidad es necesaria para la identificación de las prácticas vulnerables a la corrupción. Dicho diagnóstico no debe limitarse a las tipologías de actos de corrupción en el código penal o las especificadas como faltas administrativas del funcionario público, sino que debe partir de las características propias de las funciones de cada entidad, así como aquellas prácticas irregulares que son detectadas por la ciudadanía (Yamada y Montero, 2011). Por ello, se debe constituir diagnósticos que precisen las áreas más expuestas a riesgos de corrupción, el tipo de práctica que se realiza y los actores que están involucrados, a través de la recolección de información de las áreas afectadas. Como se mencionó anteriormente, si una política institucional anticorrupción no se vincula con los problemas y necesidades de cada entidad, solo se convierte en un incremento de burocracia o en «soluciones» sin impacto real. 


\section{REFERENCIAS}

Aguilar, L. (1993). La implementación de las politicas públicas. México: Miguel Ángel Porrúa.

Bardales, S. (2010). Delitos aduaneros de mayor incidencia en Tumbes. Lima: Fiscalía Provincial Especializada en Delitos Aduaneros y Contrabando.

CAN (2014). Lineamientos para la Elaboración de Planes Institucionales Anticorrupción. Lima: CAN-PCM.

Chêne, M. (2009). Coordination Mechanisms of AntiCorruption Institutions. Noruega: U4 Anticorruption Resource Centre.

El Comercio (2009). Empleados denunciados por corrupción siguen en la Sunat por fallos judiciales. http://elcomercio.pe/sociedad/lima/empleados-denunciados-corrupcion-siguen-Sunat-fallosjudiciales-noticia-327640

Cortázar, J. C. (2007). Entre el diseño y la evaluación. El papel crucial de la implementación de los programas sociales. New York: BID.

Garnier, L. (2000). Función de coordinación de planes y políticas. Santiago de Chile: Instituto Latinoamericano y del Caribe de Planificación Económica y Social, ILPES.

Heilbrunn, J. (2004). Anticorruption Commissions: Panacea or real medicine to fight corruption? Washington, D.C Banco Mundial. Recuperado de http://web.worldbank.org/archive/website 01006/WEB/IMAGES/WBI37234.PDF

Hussmann, K. (2007). Anti-corruption policy making in practice: What can be learned for implementing Article 5 of UNCAC? Vol. 1. Noruega: Chr. Michelsen Institute.

Martínez, R. (2010). La coherencia y la coordinación de las políticas públicas. Aspectos conceptuales y experiencias. En Proyecto de Modernización del Estado (ed.), Los desafíos de la coordinación y la integralidad de las políticas y gestión públicas en América Latina. Buenos Aires: Jefatura de Gabinete de Ministros.

Molina, C. y Licha, I. (2005). Coordinación de la politica social: criterios para avanzar. Paper presented at the X Congreso Internacional del CLAD sobre la Reforma del Estado y de la Administración Pública, Santiago.

Mujica, J. (2013). Problemas y consideraciones básicas sobre la medición del delito en el Perú. En Desco (ed.), Perú Hoy: El Perú subterráneo. Lima: Desco.

Oferlccor (2014). Determinación de las áreas que presentan mayor riesgos de corrupción en Sunat. Lima: Sunat.

ONU (2003). Convención de las Naciones Unidas contra la Corrupción. Nueva York: ONU Oficina contra la Droga y el Delito.

Parker, N., Berthin, G., Michelle, R. y Mizrahi, Y. (2004). La corrupción en América Latina: Estudio analitico basado en una revisión bibliográfica y entrevistas. Washington: USAID.

Painter, M (1981). Central Agencies and the coordination principle. Australian Journal of Public Administration, 40(4). Canberra: Institute of Public Administration Australia. https://doi. org/10.1111/j.1467-8500.1981.tb00519.x

Pautrat, L. (2016). Politicas anticorrupción. Lima: CIES.

Peters, G. (1998). Managing horizontal government. The Politics of coordination. Public Administration, 76(2), 295-311. Oxford: Blackwell Publishers Ltd. https://doi.org/10.1111/1467-9299.00102

Peters, G. (2013). Toward Policy Coordination: alternatives to hierarchy. Policy and Politics, 41(4), 569-584. https://doi.org/10.1332/030557312X655792

Peñailillo, M. (2011). Programas Anticorrupción en América Latina y el Caribe. Estudios sobre tendencias anticorrupción y proyectos del PNUD. Panamá: PNUD. Centro Regional para América Latina y el Caribe. 
PNUD (2013). Hacia una primera reflexión sobre politicas anticorrupción: Algunos insumos para la implementación de la Convención de las Naciones Unidas contra la Corrupción desde la experiencia de América Latina. Panamá: PNUD Centro Regional para América Latina y el Caribe.

Repetto, F. (2010). Coordinación de políticas sociales: abordaje conceptual y revisión de experiencias latinoamericanas. En Proyecto de Modernización del Estado (Ed.), Los desafíos de la coordinación y la integralidad de las politicas y gestión pública en América Latina (pp. 47-81). Buenos Aires: Jefatura del Gabinete de Ministros.

Repetto, F. y Fernández, J. (2012). Coordinación de politicas, programas y proyectos sociales. Buenos Aires: Fundación CIPPEC.

Silva, S. (2012). La Comisión de Alto Nivel Anticorrupción CAN como eje articulador en el desarrollo de una cultura anticorrupción. En J. M. Simon y W. Ramírez (eds.), La lucha contra la corrupción en el Perú. El modelo peruano: La experiencia de las Procuradurías Anticorrupción. Lima: Ministerio de Justicia y Derechos Humanos/Cooperación Alemana para el Desarrollo Internacional GIZ.

Yamada, G. y Montero, R. (2011). Corrupción e inequidad en los servicios públicos en Perú. Lima: Universidad del Pacífico, CIES. 